\title{
A theoretical model of a new electrostatic transducer incorporating fluidic amplification
}

\author{
Alan J. Walker \\ and Anthony J. Mulholland \\ Department of Mathematics \\ University of Strathclyde \\ Glasgow, United Kingdom, G1 1XH \\ Email: ra.awal@maths.strath.ac.uk \\ ajm@maths.strath.ac.uk
}

\author{
Ewan Campbell \\ and Gordon Hayward \\ Centre for Ultrasonic Engineering \\ Department of Electronic and Electrical Engineering \\ University of Strathclyde \\ Glasgow, United Kingdom, G1 1XW \\ Email: ewancampbell@eee.strath.ac.uk \\ g.hayward@eee.strath.ac.uk
}

\begin{abstract}
This article concerns the design of a new electrostatic transducer whose backplate consists of a series of drilled pipes. A new one-dimensional model is derived which considers the interaction of the membrane with the air load, the air cavities, and the drilled pipes in the backplate. Dynamic equations for the impedance in each component of the device are calculated analytically and connected using interface conditions of continuity of pressure and radiation conditions into the air load. The model is able to produce solutions to the mechanical impedance of the device and the displacement of the membrane as a function of the device's design parameters. Model results for the output pressure compare well with previous experimental data. The inverse problem of retrieving the design parameters for a desired output is discussed.
\end{abstract}

\section{INTRODUCTION}

Electrostatic ultrasonic transducers, or capacitive micromachined ultrasonic transducers (CMUTs), are used for the detection and generation of ultrasonic waves [1]. These transducers consist of a thin membrane stretched across a conducting backplate, which is often rough or grooved. This article considers a one-dimensional model of an electrostatic transducer whose backplate consists of a series of drilled pipes, proposed by Campbell et al. [2]. These pipes act much like tubular sections in musical instruments which amplify the sound produced. Figure 1 provides a schematic of the transducer design to be modelled. The figure describes a dielectric membrane stretched over a backplate which consists of many cavities with each cavity being connected to an air conduit. To reduce the complexity of the model we will consider only one cavity, yet retain the possibility of one or more pipes per cavity. The output of the model is then compared with the experimental results [2] in terms of the pressure transmitted by the membrane into an air load. Output pressure profiles are provided for changes in the design parameters of the model which indicate the possibility of automating the choice of design parameters given a desired output.

\section{MODEL}

We start by adapting the three-dimensional CMUT model proposed by Caronti et al. [3] to include the effects of the air load on both sides of the membrane. We reduce the spatial

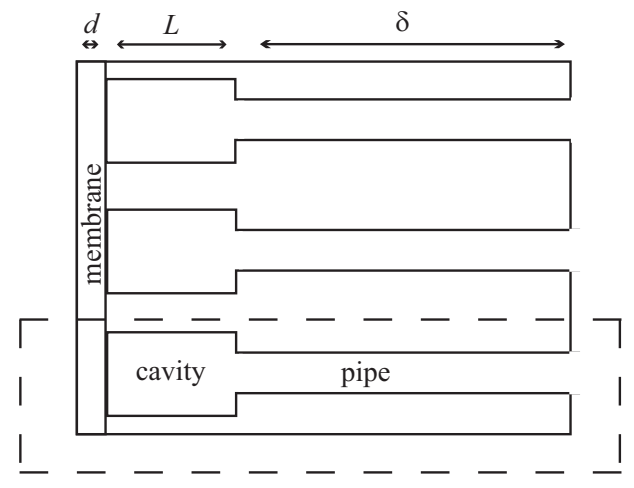

Fig. 1. Schematic diagram of the CMUT device being investigated where the dashed box indicates the specific part of the device modelled in section II.

dimensionality to unity by assuming that the membrane acts in a piston like manner. We therefore propose the following model for the displacement of the membrane

$$
\begin{aligned}
d_{m} \rho_{s} \frac{d^{2} \xi}{d t^{2}}+\frac{d \xi}{d t} & \left(R_{v}+\frac{Z_{c m}}{S}+\frac{Z_{m}}{S}\right) \\
& +\xi\left(\frac{\rho_{a} c^{2} S}{V_{0}}-\frac{\epsilon_{0} V_{D C}^{2}}{d^{3}}\right)=f(t),
\end{aligned}
$$

where $d_{m}$ is the membrane thickness, $\rho_{s}$ is the membrane mass density, $\xi=\xi(t)$ is the displacement of the membrane, $R_{v}$ is a viscous damping term associated with energy loss in the membrane, $Z_{c m}$ is the impedance of the air load at the 'cavity' side of the membrane, $S$ is the cross-sectional area of the membrane, $Z_{m}$ is the impedance of the air load at the 'open' side of the membrane, $\rho_{a}$ is the equilibrium density of air, $c$ is the speed of sound in air, $V_{0}$ is the volume of the cavity, $\epsilon_{0}$ is the permittivity of free space, $V_{D C}$ is the bias voltage applied to the membrane, $d=L+d_{m} / \epsilon_{r}$ is the electrode spacing, $L$ is the length of the cavity, $\epsilon_{r}$ is the relative permittivity of the membrane and $f(t)$ is the voltage driving force applied to the membrane. Using the standard results for pipe-driver systems [4, p.272], the impedance $Z_{c m}$ can be calculated in terms of the design parameters of the 
device, that is,

$$
Z_{c m}=\frac{\rho_{a} c S\left(Z_{c L}+j \rho_{a} c S \tan (k L)\right)}{\rho_{a} c S+j Z_{c L} \tan (k L)},
$$

where $k$ is the wavenumber (complex to include the viscous loss in air) and $Z_{c L}$ is the impedance at the cavity/pipe intersection. This is given by

$$
Z_{c L}=\frac{\rho_{a} c \gamma\left(Z_{p \delta}+j \rho_{a} c \gamma \tan (k \delta)\right)}{N\left(\rho_{a} c \gamma+j Z_{p \delta} \tan (k \delta)\right)},
$$

where $\gamma$ is the cross-sectional area of the pipe(s), $N$ is the number of pipes per cavity, $\delta$ is the length of the pipe(s) and $Z_{p \delta}$ is the impedance at the pipe/air interface. We assume in the following that all pipes are identical but the general model can cope with a distribution of pipe dimensions. The impedance $Z_{p \delta}$ is given by [4, p.186]

$$
Z_{p \delta}=\frac{1}{2} \frac{\rho_{a} c \gamma^{2} k^{2}}{\pi}+j \frac{8}{3} \frac{\rho_{a} c \gamma^{\frac{3}{2}} k}{\pi^{\frac{3}{2}}} .
$$

Similarly, the impedance $Z_{m}$ is given by

$$
Z_{m}=\frac{1}{2} \frac{\rho_{a} c S^{2} k^{2}}{\pi}+j \frac{8}{3} \frac{\rho_{a} c S^{\frac{3}{2}} k}{\pi^{\frac{3}{2}}} .
$$

Here it is assumed that we operate in the low frequency limit, that is $k S \ll 1$ and $k \gamma \ll 1$.

In order to compare the model with the experimental data provided by Campbell et al. [2], we seek the velocity (and hence the pressure output) of the membrane which can be found via Fourier transforms. Additionally, the forcing function used in the experiments $f(t)$ is a single-cycle sinusoid centred at $7.7 \mathrm{kHz}$ [2]. That is

$$
f(t)=\frac{\epsilon_{0} V_{D C} V}{d^{2}} e^{j \omega t} e^{-\frac{(t-a)^{2}}{b^{2}}},
$$

where $V$ is the excitation voltage, $\omega$ is the wavenumber and $a$ and $b$ are constants which determine the position and number of cycles in the time domain.

The pressure in the cavity $P_{c}$ is given by

$$
P_{c}=a_{c} e^{j(\omega t+k(L-x))}+b_{c} e^{j(\omega t-k(L-x))},
$$

where $a_{c}$ and $b_{c}$ are arbitrary constants which are calculated by imposing continuity of pressure at each interface and the radiation conditions (4) and (5). A similar form for the pressure in the cavity $P_{p}$ can be found.

\section{RESUlTS}

Using Fourier transforms [5, p.28], the frequency domain impulse response of equation (1) is $-1 /(j \omega Z(\omega))$ where

$$
\begin{aligned}
Z(\omega)=-j d_{m} \rho_{s} \omega+ & R_{v}+\frac{Z_{c m}}{S}+\frac{Z_{m}}{S} \\
& +\frac{j}{\omega}\left(\frac{\rho_{a} c^{2} S}{v_{0}}-\frac{\epsilon_{0} V_{D C}^{2}}{d^{3}}\right) .
\end{aligned}
$$

By convolving the impulse response with the forcing function and using the convolution theorem, the frequency domain response of the device is given by $\Xi(\omega)=-F(\omega) / Z(\omega)$, where $F(\omega)$ is the Fourier transform of the forcing function $f(t)$. The

\begin{tabular}{|c|c|c|c|}
\hline Design Parameter & Symbol & Magnitude & Dimensions \\
\hline Density of Membrane & $\rho_{s}$ & 1392 & $\mathrm{~kg} / \mathrm{m}^{3}$ \\
Speed of Sound in Air & $c$ & 343 & $\mathrm{~m} / \mathrm{s}$ \\
Length of Pipe(s) & $\delta$ & $22 \times 10^{-3}$ & $\mathrm{~m}$ \\
Number of Pipes per Cavity & $N$ & 1 & - \\
Length of Cavity & $L$ & $10^{-3}$ & $\mathrm{~m}$ \\
Thickness of Membrane & $d_{m}$ & $5 \times 10^{-5}$ & $\mathrm{~m}$ \\
Damping Coefficient & $R_{v}$ & $10^{2}$ & $\mathrm{~kg} / \mathrm{ms}$ \\
Permittivity of Free Space & $\epsilon_{0}$ & $8.85 \times 10^{-12}$ & $\mathrm{~F} / \mathrm{m}$ \\
DC Voltage & $V_{D C}$ & 450 & $\mathrm{~V}$ \\
Density or Air & $\rho_{a}$ & 1.2 & $\mathrm{~kg} / \mathrm{m}^{3}$ \\
Membrane Dielectric Constant & $\epsilon_{r}$ & 3.2 & - \\
Magnitude of AC Voltage & $V$ & 1 & $\mathrm{~V}$ \\
Constant & $a$ & $2 \times 10^{-4}$ & $\mathrm{~s}$ \\
Constant & $b$ & $5 \times 10^{-5}$ & $\mathrm{~s}$ \\
Aspect Ratio of Cavity & $\alpha_{c}$ & 1.5 & - \\
Aspect Ratio of Pipe & $\alpha_{p}$ & $5 \times 10^{-2}$ & - \\
\hline
\end{tabular}

TABLE I

DESIGN PARAMETER VALUES FOR THE CMUT MODEL.

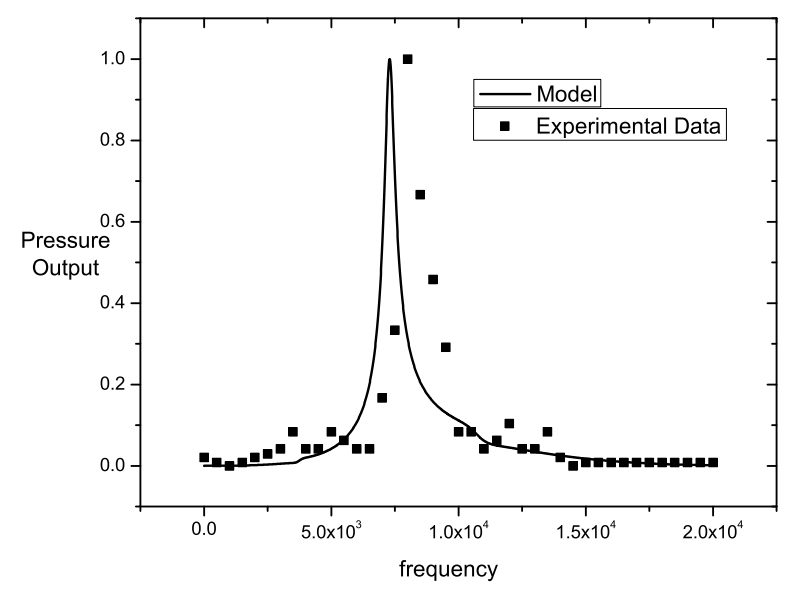

Fig. 2. Plot showing experimental data from [2] (squares) and the CMUT model described above (solid line). Here, the pressure output has been normalised for comparison purposes. We find a good agreement of results even though the effects of the microphone and electrical load have been neglected in the model.

output pressure $P_{\text {out }}$ is then given by $P_{\text {out }}=j \omega \Xi(\omega) Z_{m} / S$. We introduce two aspect ratios, $\alpha_{c}$ and $\alpha_{p}$ which describe the relationship between the length of the cavity to its radius, and length of the pipe to its radius, respectively. That is, $S=\pi\left(\alpha_{c} L\right)^{2}$ and $\gamma=\pi\left(\alpha_{p} \delta\right)^{2}$. In figure 2 the model output is compared with experimental data presented in [2]. The agreement is very good especially when we consider that the effects of the microphone and the electrical load are neglected in the model. Here, and in every plot herein, the parameter values stated in table I are used, unless instructed otherwise.

In order to investigate which design parameters significantly affect the device performance, three-dimensional plots are constructed which plot the pressure as a function of several design parameters: the length of the pipe $\delta$, the length of the cavity $L$, the thickness of the membrane $d_{m}$, the aspect ratio of the cavity $\alpha_{c}$ and the aspect ratio of the pipe $\alpha_{p}$. 


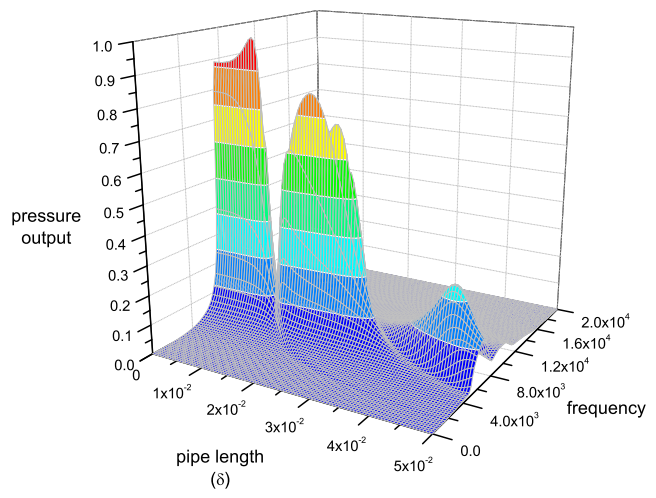

Fig. 3. Normalised output pressure plotted against frequency and pipe length.

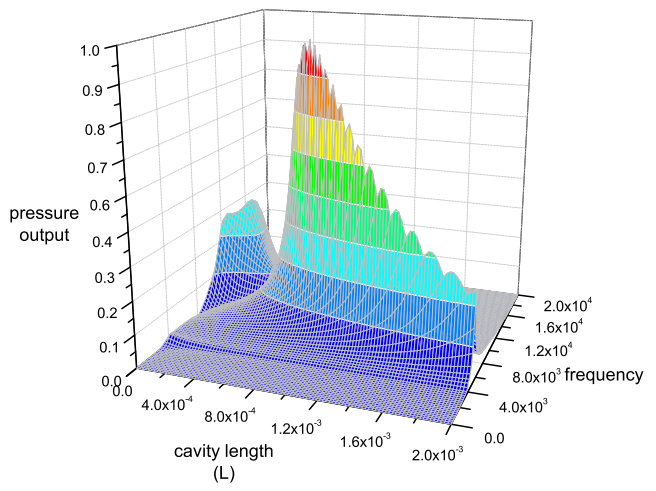

Fig. 4. Normalised output pressure plotted against frequency and cavity length.

These plots are found in figures 3, 4, 5, 6 and 7, respectively. Here, all design parameters are as stated in table I unless being varied. It can be seen that each of these design parameters can significantly affect the output pressure.

Figure 3 shows the dependence of the device on the occurrence and length of pipes. It is quite clearly seen that incorporating pipes into the device significantly increases the pressure output. Also, optimal pipe lengths can be seen, providing evidence of being able to optimise the devise for a maximum pressure output.

Figure 4 shows the dependence of the device on the length of the cavity. Similarly to the results shown for the pipe length, we can see that the occurrence of cavities increases the pressure output and an optimal cavity length can be found.

The dependence of the device on the membrane thickness can be seen in figure 5. Again, optimal membrane thickness can be calculated from the results presented.

It can be seen from figures 6 and 7 that the ratio of the radius to the length of the cavity (and the pipe) does not significantly affect the pressure output. The change in output pressure seen in figure 6 can be attributed to the rising surface area of the membrane.

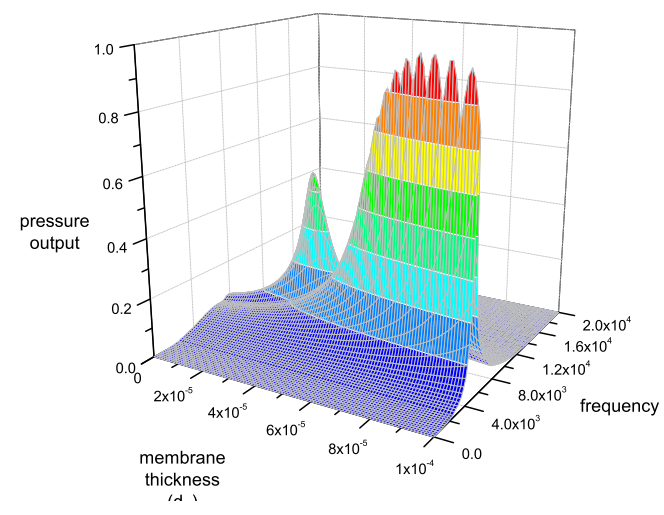

Fig. 5. Normalised output pressure plotted against frequency and membrane thickness.

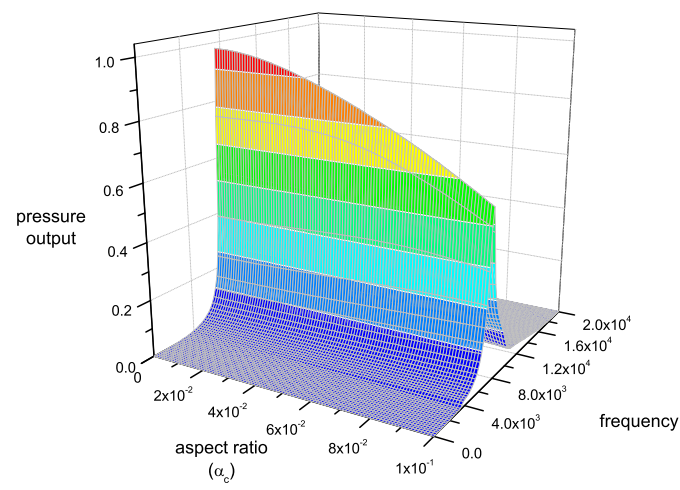

Fig. 6. Normalised output pressure plotted against frequency and aspect ratio of cavity.

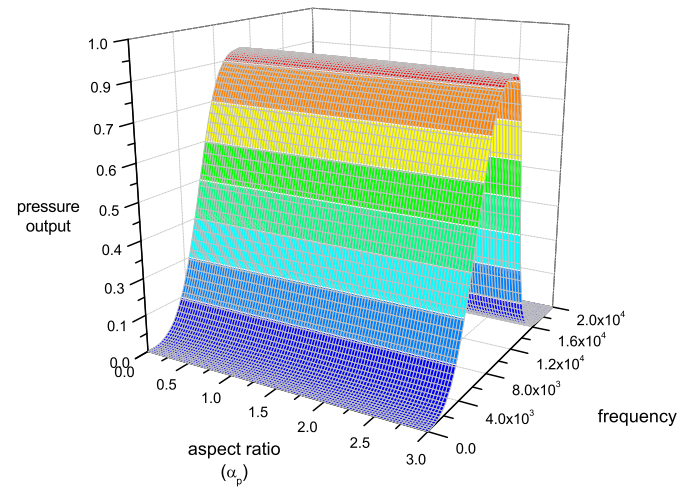

Fig. 7. Normalised output pressure plotted against frequency and aspect ratio of pipe.

\section{CONCLUSION}

This article has presented an analytical theory to model the resonant behaviour of a capacitive michromachined ultrasound transducer (CMUT) incorporating fluidic amplification. The results of the model compared well with the experimental data and the model also allows us to investigate the dependence 
of the device performance on the design parameters. This modelling approach can also be extended to model more than one cavity, with the possibility of more than one pipe per cavity. In doing so, a desired pressure output profile could be realised by varying the size distribution of the pipes. This inverse problem is the subject of ongoing investigations.

\section{ACKNOWLEDGMENT}

The authors would like to acknowledge the support of the UK Engineering and Physical Sciences Research Council (EPSRC) (Grant EP/F017421/1).

\section{REFERENCES}

[1] W. Kuhl, R. Schodder, and F. K. Schroder, "Condenser transmitters and microphones with solid dielectric diaphragms for airborne ultrasonics," Acustica, vol. 4, pp. 520-532, 1954.

[2] E. Campbell, W. Galbraith, and G. Hayward, "A new electrostatic transducer incorporating fluidic amplification," IEEE Ultrasonics Symposium, pp. 1445-1448, 2006.

[3] A. Caronti, G. Caliano, A. Iula, and M. Pappalardo, "An accurate model for capacitive micromachined ultrasonic transducers," IEEE transactions on ultrasonics, ferroelectrics, and frequency control, vol. 49, 2002.

[4] L. E. Kinsler, A. R. Frey, A. B. Coppens, and J. V. Sanders, Fundamentals of Acoustics. John Wiley \& Sons, inc., 2005.

[5] P. M. Morse and K. Uno Ingard, Theoretical Acoustics. Princeton University Press, 1968. 